\title{
Theoretical Modeling for Hepatic Microwave Ablation
}

\author{
Punit Prakash*
}

Department of Radiation Oncology, University of California, San Francisco, USA

\begin{abstract}
Thermal tissue ablation is an interventional procedure increasingly being used for treatment of diverse medical conditions. Microwave ablation is emerging as an attractive modality for thermal therapy of large soft tissue targets in short periods of time, making it particularly suitable for ablation of hepatic and other tumors. Theoretical models of the ablation process are a powerful tool for predicting the temperature profile in tissue and resultant tissue damage created by ablation devices. These models play an important role in the design and optimization of devices for microwave tissue ablation. Furthermore, they are a useful tool for exploring and planning treatment delivery strategies. This review describes the status of theoretical models developed for microwave tissue ablation. It also reviews current challenges, research trends and progress towards development of accurate models for high temperature microwave tissue ablation.
\end{abstract}

\section{INTRODUCTION}

Thermal ablation is the destruction of tissue in situ using heat. In this technique, devices deliver energy to raise tissue to lethal temperatures (above $\sim 50-60{ }^{\circ} \mathrm{C}$ ). Many energy sources, including laser, microwave, radiofrequency currents, thermal conduction and high intensity ultrasound, have been used for performing ablation. Cryoablation is another form of thermal ablation that involves freezing to cause cell death. These techniques have been used in diverse medical applications including treatment of cancer and benign tumors, cardiac arrhythmias, tissue reshaping and others [1]. Several reviews of the different energy modalities for thermal ablation of tissue are available in the literature [1-4]. While radiofrequency ablation (RFA) [5] and cryoablation [6] have been the most clinically used modalities for thermal ablation of hepatic tumors, microwave ablation (MWA) is a promising technology that offers several advantages to these techniques. Advantages of using microwaves over other energy modalities for thermal tumor ablation have been reviewed elsewhere [3, 7, 8]. Briefly, microwaves enable faster heating of larger targets, induce higher temperatures within tissue, are less susceptible to perivascular heat sinks and do not require the use of ground pads. In this review, we focus on the use of MWA for treatment of liver tumors. MWA is also being explored for treating tumors in kidney [9, 10], lung [11-13], bone [14, 15], prostate [16, 17] and other tumors [18], as well as for treatment of cardiac arrhythmias [19]. Lastly, microwave energy has also been used for regional hyperthermia (moderate elevation of tissue temperatures to $\sim 41-45{ }^{\circ} \mathrm{C}$ ), which is typically used as an adjuvant to radiotherapy and/or chemotherapy [20, 21].

During MWA, an antenna (also referred to as applicator or device) is inserted into the target organ either percutaneously, laparoscopically or under open surgery, with ultrasound or CT guidance. Several antennas have been

*Address correspondence to this author at the Department of Radiation Oncology, University of California, San Francisco, USA;

E-mail: punit.prakash@gmail.com developed for microwave tissue ablation, such as the slot antenna [22, 23], cap-choke antenna [24], floating sleeve antenna [25], and triaxial antenna [26, 27], and have been reviewed in [28]. Microwave energy, typically at $915 \mathrm{MHz}$ or $2.45 \mathrm{GHz}$, radiated by the antenna causes rapid rotation of water, proteins and other polar molecules in tissue, a process known as dielectric hysteresis. This in turn leads to heating of the tissue. Cell death is a function of elevated temperature and the duration for which the elevated temperature is maintained [29]. At temperatures exceeding $60{ }^{\circ} \mathrm{C}$, cell death is almost instantaneous [30]. Typical treatment durations are on the order of 3 to $12 \mathrm{~min}$ [2].

For treatment of cancer, the goal of thermal ablation mechanisms is to create an ablation zone that extends $1 \mathrm{~cm}$ beyond the tumor boundary at all points [31]. The $1 \mathrm{~cm}$ safety margin is used in order to ensure complete destruction of the entire tumor and to minimize chances of recurrence. The extent of the ablation zone is determined by the timetemperature profile created in tissue by the supplied electromagnetic energy [32]. Thus, there is a need for techniques to accurately predict and measure the transient temperature profile in tissue during ablation treatments.

Theoretical models of MWA solve the partial differential equations (PDEs) governing electromagnetic wave propagation and heat transfer in tissue to determine the resultant temperature change during an ablation procedure. Due to the temperature dependence of tissue physical properties, these problems are inherently coupled. Analytical methods are rarely applicable because of complex device geometries and nonlinear tissue properties. Instead, numerical techniques are frequently employed to solve the relevant PDEs with the aid of computers. While in vivo animal studies and clinical trials are the ultimate test for evaluating devices, theoretical models play a crucial role during the design of devices for MWA - serving as a quick, convenient and inexpensive evaluation to isolate and optimize promising devices for prototyping. They also serve as a means for understanding the interaction between the various physical phenomena that occur during ablation treatments. Lastly, theoretical models are being explored for 
use in patient-specific treatment planning to improve the efficacy of thermal ablation therapies.

This paper reviews progress in the field of theoretical modeling of microwave tissue ablation. The rest of this paper is organized as follows. First, we describe the physical phenomena that occur within tissue upon application of electromagnetic energy. Next, we describe methods for modeling these phenomena using numerical techniques. We discuss challenges specific to modeling high temperature microwave ablation. Finally, we conclude with a review of current research trends towards developing improved theoretical models for microwave tissue ablation. We limit the scope of this paper to oncologic applications and specifically to treatment of liver tumors, which has been the most widely reported site for MWA.

\section{PHYSICAL PHENOMENA DURING MICROWAVE TISSUE ABLATION}

The ultimate goal of computer models for microwave tissue ablation is to predict the induced tissue damage. Since tissue damage is a function of the time-temperature history, accurate prediction of the temperature profile in tissue is necessary. The temperature profile in tissue during an ablation procedure is largely defined by two physical phenomena: (1) interaction of microwaves with tissue and (2) heat transfer in tissue. This section reviews microwave absorption and heat transfer in tissue, as well as a synopsis of tissue damage due to elevated temperatures.

\section{Microwave Absorption in Tissue}

The propagation and absorption of microwaves in tissue is governed by Maxwell's equations, stated in equations (1) $-(4)$.

$$
\begin{aligned}
& \nabla \cdot \mathbf{D}=\rho_{\text {free }} \\
& \nabla \cdot \mathbf{B}=0 \\
& \nabla \times \mathbf{E}=-\frac{\partial \mathbf{B}}{\partial t} \\
& \nabla \times \mathbf{H}=\mathbf{J}+\frac{\partial \mathbf{D}}{\partial t}
\end{aligned}
$$

where $\mathbf{D}\left[\mathrm{C} / \mathrm{m}^{2}\right]$ is electric flux density, $\mathbf{B}[\mathrm{T}]$ is magnetic field, $\mathbf{E}[\mathrm{V} / \mathrm{m}]$ is electric field strength, $\mathbf{H}[\mathrm{A} / \mathrm{m}]$ is magnetic field intensity, $\rho_{\text {free }}\left[\mathrm{C} / \mathrm{m}^{2}\right]$ is free charge density, and $\mathbf{J}$ $\left[\mathrm{A} / \mathrm{m}^{2}\right]$ is current density. The electromagnetic fields radiated in tissue by a given antenna can be determined by solving Maxwell's equations, given knowledge of tissue electromagnetic properties (permittivity and conductivity) and appropriate initial and boundary conditions. Therefore, it is of critical importance to characterize the relevant electromagnetic properties of tissue and how they change during the course of an ablation, in order to develop accurate models of the ablation process.

The absorption of electromagnetic power in tissues is a function of their material properties-dielectric permittivity, $\varepsilon \quad[\mathrm{F} / \mathrm{m}]$ and conductivity $\sigma[\mathrm{S} / \mathrm{m}]$. The (effective) conductivity, $\sigma$, of a material is given by $\sigma=\sigma_{\mathrm{s}}+\sigma_{\mathrm{a}}$ where $\sigma_{\mathrm{s}}$ and $\sigma_{\mathrm{a}}$ are the static and alternating (also referred to as dielectric) conductivities, respectively. Static conductivity accounts for losses at dc, while alternating conductivity accounts for losses when the material is subjected to alternating fields. Absorption of electromagnetic power in a material is often characterized by the material's loss tangent, which is defined as $\sigma / \omega \varepsilon$, where $\omega[\mathrm{rad} / \mathrm{s}]$ is angular frequency. If $\sigma / \omega \varepsilon<<1$, then the material is considered to be a low loss material, one that does not absorb much of the electromagnetic energy propagating though it. Materials with $\sigma / \omega \varepsilon \sim 1$ are referred to as lossy, and absorb substantial electromagnetic energy as waves propagate through them. The permittivity and conductivity of a material may be lumped together into a single, complex valued number known as its complex permittivity given by:

$$
\varepsilon_{\mathrm{r}}=\varepsilon_{\mathrm{r}}^{\prime}-j \frac{\sigma}{\omega \varepsilon_{0}}
$$

where $\sigma[\mathrm{S} / \mathrm{m}]$ is the effective conductivity, $\varepsilon_{\mathrm{r}}{ }^{\prime}[\mathrm{F} / \mathrm{m}]$ is the real relative permittivity of the material and $\varepsilon_{0}[\mathrm{~F} / \mathrm{m}]$ is the permittivity of free space.

Most organ tissues are lossy, with alternating conductivity much larger than static conductivity. In soft tissue, both $\sigma$ and $\varepsilon$ vary with frequency, temperature and water content. Below, we review several methods for characterizing changes in tissue dielectric properties as a function of frequency, temperature and water content.

The Cole-Cole model is the most widely used method for specifying frequency-dependent complex permittivity of tissue. The Cole-Cole equation is:

$$
\varepsilon(f)=\varepsilon_{\infty}+\sum_{n} \frac{\varepsilon_{\mathrm{s}}-\varepsilon_{\infty}}{1+\left(j 2 \pi f \tau_{n}\right)^{1-\alpha_{n}}}+\frac{\sigma_{\mathrm{i}}}{2 \pi \varepsilon_{0}}
$$

where $\varepsilon_{\infty}$ is the relative permittivity at infinite frequency, $\varepsilon_{\mathrm{s}}$ is the relative permittivity at dc, $f[\mathrm{~Hz}]$ is frequency, $\tau_{n}$ is the $n^{\text {th }}$ relaxation time constant, $\alpha_{n}$ is an attenuation constant and $\sigma_{\mathrm{i}}[\mathrm{S} / \mathrm{m}]$ is dc conductivity. Relaxation from multiple frequency bands can be incorporated with the use of $n$ relaxation terms in (6). Cole-Cole model parameters have been determined for many different soft tissues and are readily available in the literature $[33,34]$.

A few studies have reported dielectric properties of tumor and normal liver tissues. Stauffer et al. [35] measured dielectric properties of excised samples of primary liver cancer (hepatocellular carcinoma), liver metastases (metastatic colorectal carcinoma) and normal liver tissue from human subjects. Relative permittivity and conductivity of liver tumors were found to be $12 \%$ and $24 \%$ higher than normal liver tissue [35]. Their study also showed that properties of ex vivo bovine tissue, a commonly used model for evaluating prototype devices, are similar to that of normal liver tissue. O'Rourke et al. [36] measured wideband dielectric properties of normal and malignant human liver tissues, both in vivo and ex vivo. Results of this study agreed with [35], showing that relative pertmittivity and conductivity of malignant tissue is higher than that of normal tissue, when measured ex vivo. However, for measurements made in vivo, they reported no statistically significant differences in tissue dielectric properties. Further studies to characterize dielectric properties of tissue in vivo are warranted. 
Dielectric properties of tissue are also dependent on temperature. This is mainly due to temperature-dependent dielectric property changes of tissue water and proteins. At temperatures above $60{ }^{\circ} \mathrm{C}$, when protein denatures, changes in tissue dielectric properties are irreversible. Coefficients of dielectric property changes as a function of temperature (up to $60{ }^{\circ} \mathrm{C}$ ) have been determined from measured data [34, 37]. Relative permittivity decreases slightly with temperature - $0.2 /{ }^{\circ} \mathrm{C}$ at $915 \mathrm{MHz}$ and $-0.1 /{ }^{\circ} \mathrm{C}$ at $2.45 \mathrm{GHz}[34,37]$. Conductivity increases slightly with temperature $-1.33 /{ }^{\circ} \mathrm{C}$ at $915 \mathrm{MHz}$ and $0.4 /{ }^{\circ} \mathrm{C}$ at $2.45 \mathrm{GHz}[34,37]$. While specifying temperature coefficients for dielectric properties has been widely used, other approaches have also been suggested. For instance, Lazebnik et al. [37] measured wideband dielectric properties of tissue at temperatures up to $60{ }^{\circ} \mathrm{C}$, and determined coefficients for a polynomial fit to the temperature-dependent Cole-Cole parameters. Measurements of tissue dielectric properties at temperatures in excess of $60{ }^{\circ} \mathrm{C}$ are scarce. Brace [38] reported measurements of tissue dielectric properties at $915 \mathrm{MHz}$ and $2.45 \mathrm{GHz}$ taken during $\mathrm{RF}$ ablation of ex vivo liver tissue, showing a sharp fall (up to 50\%) in relative permittivity and conductivity at temperatures near $100{ }^{\circ} \mathrm{C}$. This was likely due to tissue water vaporization at these temperatures. When heating was stopped, measured dielectric properties at lower temperatures were much lower than their original values, indicating irreversible damage [38]. Further studies of dielectric properties are warranted at elevated temperatures up to $150{ }^{\circ} \mathrm{C}$, which are routinely achieved during MWA [39]. At elevated temperatures, it is difficult to isolate changes in dielectric properties solely as a function of temperature, as changes due to water vaporization and subsequent tissue desiccation are dominant. Ultimate, a method for characterizing tissue dielectric properties as a function of thermal dose is desirable.

Changes in tissue dielectric properties have also been characterized as a function of their water content [40] (see Table 1). At steady-state, liver tissue is approximately $78 \%$ water by mass. At temperatures above $100{ }^{\circ} \mathrm{C}$, tissue water evaporates and tissue water content may decrease to less than $20 \%$ by mass. This results in drastic changes in tissue dielectric parameters. Since a large fraction of liver tissue consists of water, the dielectric properties of liver tissue are similar to those of water. As water evaporates, liver tissue becomes less lossy, which results in greater penetration of microwaves. Consequently, the radiation pattern and impedance matching of the antenna may change substantially during the course of an ablation.

\section{Heat Transfer in Tissue}

The temperature profile in tissue during ablation is obtained by solving a bioheat equation. The most widely used bioheat equation for modeling thermal therapy procedures is the Pennes bioheat equation [41]:

$$
\rho c \frac{d T}{d t}=\nabla \cdot k \nabla T+Q-Q_{\mathrm{p}}+Q_{\mathrm{m}}
$$

where $\rho\left[\mathrm{kg} / \mathrm{m}^{3}\right]$ is mass density, $c[\mathrm{~J} / \mathrm{kg} \cdot \mathrm{K}]$ is specific heat capacity, $k[\mathrm{~W} / \mathrm{m} \cdot \mathrm{K}]$ is thermal conductivity, $T[\mathrm{~K}]$ is temperature, $Q\left[\mathrm{~W} / \mathrm{m}^{3}\right]$ is the absorbed electromagnetic energy, $Q_{\mathrm{p}}\left[\mathrm{W} / \mathrm{m}^{3}\right]$ is the heat loss due to microvascular blood perfusion, and $Q_{\mathrm{m}}\left[\mathrm{W} / \mathrm{m}^{3}\right]$ is metabolic heat generation. The blood perfusion term, $Q_{\mathrm{p}}$, is given by (8).

$$
Q_{\mathrm{p}}=\omega_{\mathrm{bl}} c_{\mathrm{bl}}\left(T-T_{\mathrm{bl}}\right)
$$

where $\omega_{\mathrm{b}}\left[\mathrm{kg} / \mathrm{m}^{3} \cdot \mathrm{s}\right]$ is blood perfusion rate, $c_{\mathrm{bl}}[\mathrm{J} / \mathrm{kg} \cdot \mathrm{K}]$ is the specific heat capacity of blood and $T_{\mathrm{bl}}[\mathrm{K}]$ is blood temperature. Generally, $Q_{\mathrm{m}}$ is ignored as its magnitude is substantially smaller than other terms in this equation. The absorbed electromagnetic energy, $Q$, is computed from the electromagnetic field distribution in tissue, and is given by (9).

$$
Q=\frac{1}{2} \sigma|E|^{2}
$$

When normalized by tissue density, $Q$ is referred to as the specific absorption rate (SAR).

As is the case with the electromagnetic model, characterization of tissue thermal properties is essential for accurate prediction of the temperature profile. Tissue thermal properties vary as a function of temperature and water content [34].

Thermal properties of tissue - thermal conductivity, specific heat capacity, and density - have been measured by various groups and reported in the literature. Tissue density

Table 1. Empirical Formulae for Tissue Properties as a Function of their Water Content

\begin{tabular}{|l|l|l|}
\hline Parameter & Steady-State Value & Empirical Formula (and Reference) \\
\hline \hline Dielectric permittivity & $43.03[\mathrm{~F} / \mathrm{m}](@ 2.45 \mathrm{GHZ})$ & $\varepsilon=1.71 f^{-1.13}+\frac{\varepsilon_{s}^{m}-4}{1+(f / 25)^{2}}+4[40]$ \\
\hline Dielectric conductivity & $\begin{array}{l}1.69[\mathrm{~S} / \mathrm{m}] \\
(@ 2.45 \mathrm{GHz})\end{array}$ & $\sigma=1.35 f^{0.13} \sigma_{0.1}+\frac{0.0222\left(\varepsilon_{s}^{m}-4\right) f^{2}}{1+(f / 25)^{2}}[40]$ \\
\hline Thermal conductivity & $0.512[\mathrm{~W} /(\mathrm{m} \cdot \mathrm{K})]$ & $k\left(v_{w}\right)=0.603 v_{w}+0.04329+\left(0.778-v_{w}\right) \times 0.0316[34]$ \\
\hline Specific heat capacity & $3600[\mathrm{~J} /(\mathrm{kg} \cdot \mathrm{K})]$ & $k\left(v_{w}\right)=0.603 v_{w}+0.04329+\left(0.778-v_{w}\right) \times 0.0316[34]$ \\
\hline Density & $1060\left[\mathrm{~kg} / \mathrm{m}^{3}\right]$ & $\rho\left(v_{w}\right)=1000 v_{w}+288.6[34]$ \\
\hline
\end{tabular}


is typically assumed constant with temperature. For ablations where maximum temperature exceeds $100{ }^{\circ} \mathrm{C}$ and water vaporizes, tissue density should be adjusted to account for desiccation. Empirical formulae for various tissue densities as a weighted average of their principal constituents have been reported [34]. Specific heat capacity for various tissue types is available in the literature [34]. Haemmerich et al. $[42,43]$ measured specific heat of bovine liver as a function of temperature up to $\sim 85{ }^{\circ} \mathrm{C}$. Specific heat of bovine liver tissue was found to increase with temperature by up to $18 \%$ at $83.5{ }^{\circ} \mathrm{C}$. At more elevated temperatures, accurate measurement of specific heat is difficult due to sample water loss as it is heated. For ablations where peak temperatures are above $100{ }^{\circ} \mathrm{C}$, it is important to account for the latent heat associated with water vaporization - a substantial heat sink [44-47]. Thermal conductivity of various tissue types have been measured as functions of temperature, both $e x$ vivo and in vivo [48, 49]. Thermal conductivity of ex vivo bovine liver tissue increases slightly with temperature, with a coefficient of $0.00033 /{ }^{\circ} \mathrm{C}$ [48]. No data for thermal conductivity above $90{ }^{\circ} \mathrm{C}$ have been presented. To account for changes due to water vaporization, empirical formulate taking into account thermal conductivities of the principal constituents of tissue have been used [34].

The Pennes equation incorporates heat loss due to blood perfusion, modeling it as being proportional to the temperature difference between entering blood in the capillaries and local tissue. All heat transfer between tissue and blood is assumed to occur in the capillary bed. Many studies have shown this to be erroneous and more complex and rigorous models of heat transfer within tissue have been developed [50-52]. An excellent review of bioheat transfer models is provided in [53]. Nevertheless, the Pennes equation remains a remarkably effective method for modeling heat transfer in tissue during thermal ablation. Most models reported in the literature employ a constant blood perfusion up to a critical temperature - usually $60{ }^{\circ} \mathrm{C}-$ above which the perfusion term is set to zero. This is done to model the effect of collagen shrinking in vessel walls, leading to cessation of blood flow. However, experimental measurements during hyperthermia treatments have demonstrated that blood perfusion in response to elevated temperature is a complex function of temperature and time $[54,55]$. He et al. [56] modeled perfusion as initially increasing with thermal damage to tissue, before decreasing linearly with thermal damage. For hepatic RFA, Schutt and Haemmerich [57] showed that the choice of blood perfusion model substantially impacts the size and shape of ablation zone. There have been few reports of measured perfusion during ablation treatments. Moreover, due to differences in vasculature of tumor, blood perfusion within the tumor is likely substantially different from that of normal tissue. In vivo measurements of blood perfusion during thermal ablation of tumors would allow for improved modeling of this parameter.

The Pennes bioheat equation does not account for heat loss due to blood flow through large, discrete vessels - "the heat sink effect." The liver is a highly perfused organ with many large blood vessels. Ablations performed in proximity to these vessels are susceptible to the heat sink effect. Heat transfer due to blood flow in large vessels near tumors should be taken into account during planning of treatments with theoretical models. One method for doing so is to specify an effective convective heat transfer coefficient along the vessel surface $[58,59]$. A more complete analysis incorporates the complex relationship between blood flow dynamics in the vessel and transient temperature [60].

\section{Thermal Damage in Tissue}

Tissue damage due to heating is a complex function of the amount of temperature elevation as well as the duration of temperature elevation, and has been studied in great detail in the field of hyperthermia. Reviews of methods for quantifying tissue damage due to elevated temperatures are available [32, 61, 62]. Henriques and Moritz were the first to introduce kinetic models of tissue damage due to temperature $[63,64]$. The survival fraction of cells in tissue exposed to elevated temperature is given by:

$$
\Omega(t)=\ln \left\{\frac{C(0)}{C(t)}\right\}=\int_{0}^{\tau} A \exp \left(-\frac{E_{a}}{R T(\tau)}\right) d t
$$

where $C(0)$ is the original concentration of undamaged cells prior to heating, $C(t)$ is the concentration of undamaged cells after heating, $\Omega$ is a dimensionless damage parameter, $A$ $[1 / \mathrm{s}]$ is frequency factor, $E_{\mathrm{a}}[\mathrm{J} / \mathrm{mol}]$ is the activation energy required to transform tissue from normal to damaged state, $R$ $[\mathrm{J} /(\mathrm{mol} . \mathrm{K})]$ is the universal gas constant and $T[\mathrm{~K}]$ is absolute temperature history of tissue. Activation energies for various tissue types have been measured and are available in the literature [61]. Most of the measurements for these parameters have been taken for moderate elevations in tissue temperature - lower than the goal temperature for thermal ablation therapy. Further measurements of cell damage parameters at elevated temperatures for various tumor and normal tissue types are warranted, to effectively evaluate the use of kinetic models for thermal damage in tissue during ablation treatments.

Another model for cell-damage presented by Sapareto and Dewey [30] represents thermal damage with reference to equivalent time at a reference temperature (typically, $43{ }^{\circ} \mathrm{C}$ ). The time taken to achieve irreversible cell damage in cells exposed to elevated temperatures halves for each unit rise in temperature, above $43{ }^{\circ} \mathrm{C}$. Thresholds for $\Omega$ and cumulative equivalent minutes at $43{ }^{\circ} \mathrm{C}$ have been measured and correlated with experimentally observed tissue damage for various ablation modalities. Other models for cell death such as loss of birefringence [65] and heat-shock protein expression [66] have been developed.

Typically, the predicted transient temperature profile from theoretical models is compared with experimentally measured data. As ablation treatments are used more frequently in the clinic, models incorporating tissue damage will be required for predicting treatment efficacy. Critical isotherms (typically $\sim 55-60{ }^{\circ} \mathrm{C}$ ), which corresponds to visible thermal coagulation of tissue, are frequently used for evaluating ablation devices.

\section{NUMERICAL MODELS OF MICROWAVE TISSUE ABLATION}

Due to the nonlinearity of tissue properties and complex device geometries, analytical techniques are seldom used for 
solving the PDEs (1) - (4) and (7) to determine transient temperature profiles in tissue. Instead, numerical techniques such as the finite element method (FEM) and the finite difference time domain (FDTD) method are widely used to discretize the PDEs in time and space and solve for the temperature profile in tissue. Other numerical techniques such as the method of moments have also been used for solving Maxwell's equations in tissue. Many commercial tools are available for simulating tissue heating using these techniques, such as COMSOL Multiphysics, ANSYS, HFSS, and SEMCAD, while some groups have developed custom codes for simulating microwave heating of tissue. This section reviews the requirements for creating numerical models for microwave tissue ablation. It also describes MWA models of varying complexity that have been presented in the literature.

\section{Geometry}

Models that include a three dimensional definition of the antenna and target tissue are the most realistic representation of the physical problem. Typically, a simplistic geometric model of the target organ is employed for computer modeling of antenna during device design. However, some models have taken into account the geometry of target organs from imaging scans. Tissue is typically modeled as a homogenous block, ignoring the microvasculature and pores. Most models do not include distinct definitions of tumor and normal tissue. This is likely because theoretical models are usually validated in tissue phantoms and ex vivo models. Furthermore, the most frequently used in vivo animal model, porcine liver, does not incorporate a tumor. Most devices for hepatic microwave ablation are based on a coaxial design. The antenna structure is specified in fine detail so as to obtain a good approximation of its radiation pattern. Axisymmetric models can be employed to solve Maxwell's equations and the bioheat equation [67] for cylindrically symmetric coaxial designs. The antenna structure is usually specified in fine detail so as to obtain a good approximation of its radiation pattern.

\section{Boundary and Initial Conditions}

Appropriate boundary and initial conditions need to be specified for solution of the relevant PDEs. Initial tissue temperature is set to body core temperature of $37{ }^{\circ} \mathrm{C}$. The source term for the electromagnetic model needs to be carefully specified in order to launch the appropriate electromagnetic wave. Typically, for coaxial structures, azimuthal magnetic field corresponding to the intended input power is specified at one end of the coaxial cable [67]. For the electromagnetic problem, the boundary conditions along the edge of the tissue are typically specified as a perfectly matched layer (PML) or absorbing/low reflecting boundary condition to simulate an infinitely large medium. This is done in order to prevent microwave energy reflecting back into target zone from the edge of the boundary. In practice, this is negligible since the size of the tissue being modeled is typically larger than several penetration depths of microwaves. For the thermal problem, boundary conditions along the edge of the simulation space are specified as fixed temperature corresponding to body core, or as thermally insulating boundary conditions.
In order to reduce size of the simulation space, many models exclude metallic components by modeling them as perfect electric conductors (PEC). For a material with infinitely large electric conductivity, the electric field in the material goes to zero. By setting the boundary condition along the edges of all metallic components, these parts can be excluded from the computational domain. However, such models inherently ignore any losses in the coaxial cable structure. While these losses have little bearing on the radiation pattern of the antenna, they do not account for heating of the coaxial cable due to conductor and dielectric loss. Observations from experimental studies show that substantial heating of the coaxial feedline occurs during high power microwave ablation. This is likely due to dielectric and conductor loss in the coaxial feedline assembly. Therefore, accurate models should account for losses in the coaxial feedline. However, this is a challenging task since skin depth in most good conductors used for coaxial cable (e.g. copper) is very small, thus extremely fine spatial discretization is needed, which comes at the expense of increased computational burden. In the thermal model, all metallic components are readily included within the computation domain by specifying appropriate thermal properties. Fig. (1) shows a slice through an FEM model of a coaxial antenna with two ring slots.

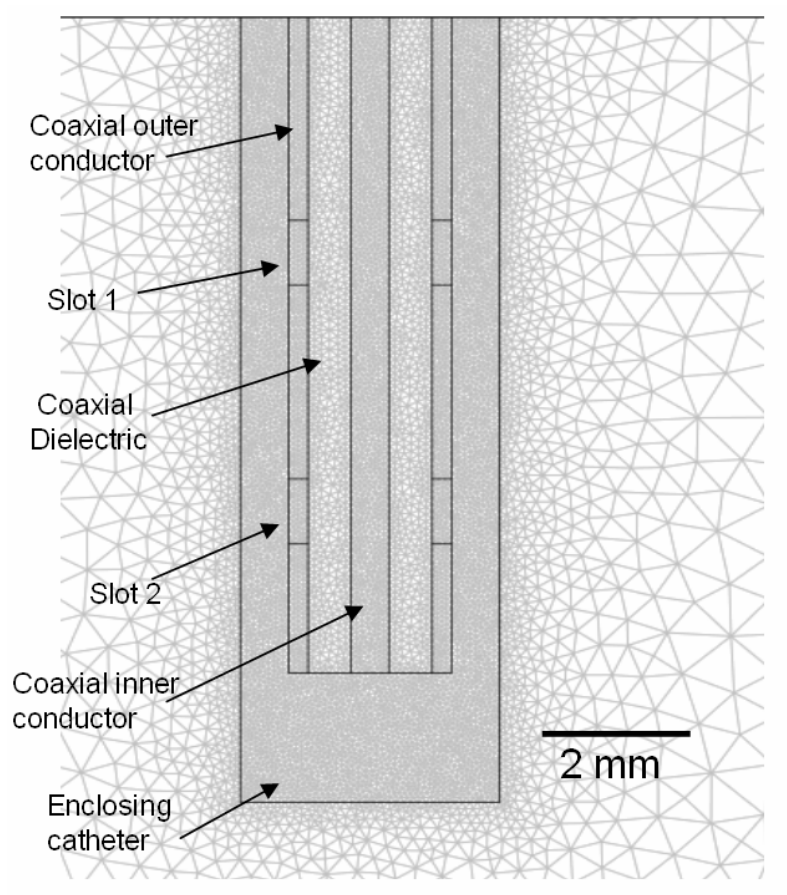

Fig. (1). Slice through a 3D finite element model of a coaxial double-slot antenna. Fine meshing $(\sim 0.1 \mathrm{~mm})$ is used in the antenna structure for accurate prediction of radiation pattern. Node spacing further away from antenna-tissue interface is on the order of $1 \mathrm{~mm}$.

\section{Spatial and Temporal Discretization}

When discretizing the computational space, wavelength in tissue is the governing factor for maximum node spacing. Typically, the maximum inter-node spacing should be an 
order of magnitude lower than the wavelength in tissue at the frequency of operation. For instance, at $2.45 \mathrm{GHz}$, the wavelength in liver tissue is $1.85 \mathrm{~cm}$. Thus, a maximum node spacing of $1 \mathrm{~mm}$ suffices. Finer spacing should be used at the source boundary, as well as boundaries between the antenna radiating element and tissue. Meshes created for solution of Maxwell's equations are more than sufficiently accurate for solution of the thermal model. Variable node spacing is usually employed to focus more computational resources in critical regions (e.g. source boundary, antenna tissue interface) and use fewer resources in regions further away from the antenna. For the FDTD method, limits on the maximum time step are governed by the Courant stability criterion [68]. Algorithms incorporating variable time-steps have been developed to speed up computation.

Although the above general guidelines work well in most cases, a Cauchy convergence test should be performed to determine whether the mesh resolution and time step size are sufficiently small. This is performed by computing some objective quantity (e.g. antenna reflection coefficient, $S_{11}$ or size of ablation zone) using the model when for increasingly finer meshes. When the change in the objective quantity due to finer mesh drops below a specified threshold, the mesh size used can be assumed to be sufficient for the problem at hand.

The following sections discuss theoretical models of various complexity for microwave tissue ablation. Some of these models are more geared towards design, optimization and testing of applicators, while others are more suitable for predicting temperature profiles induced in animal tissue models. Complexity, which is directly related to computational burden, and accuracy of the model are traded off depending on the application, when selecting a model.

\section{SAR Models}

The simplest mathematical models of devices for MWA infer tissue temperature based purely on the absorption of electromagnetic energy. Mathematically, this can be expressed as:

$$
Q=\rho c \frac{d T}{d t}
$$

This equation is obtained from (5) by neglecting terms due to metabolism, thermal conduction and perfusion. The temperature profile at any instant of time can be obtained by discretizing the time derivative in (11).

The main advantage of this model is that a steady-state solver can be used to solve Maxwell's equations to determine the resistive heating in tissue, from which temperature computation is trivial. The ability to use a steady-state solver greatly reduces computational time, making this model a particularly attractive choice in applications where a quick, but not necessarily highly accurate, prediction of the ablation zone is required. This is usually the case during model-based optimization of devices [68]. Although not highly accurate, the SAR profile in tissue provides a fair estimation of the heating profile since the direct deposition of electromagnetic energy has been shown to be the major contributing factor to heat transfer in tissue during thermal ablation [70].
SAR models are frequently used during the experimental validation of devices in tissue-mimicking phantoms [71, 72]. For this procedure, high power microwave energy is fed to the device under test for short $(<30 \mathrm{~s})$ durations. The device under test is immersed into a material with dielectric and thermal properties similar to those of biological tissue. Temperature measurements at several points are then compared to simulated temperatures, using equation (11), to determine the match between simulated and fabricated antennas. For short duration, high power ablation, thermal conductivity can be neglected and any change in phantom temperature is due to deposition of electromagnetic energy. The measured and simulated temperatures are then compared. This model is rarely used for predicting temperature profiles in ex vivo or in vivo tissue, for treatment durations longer than $30 \mathrm{~s}$.

\section{EM-Thermal Model with Static Tissue Properties}

A more realistic model than SAR models is one that incorporates thermal conductivity and blood perfusion, such as the complete Pennes bioheat equation stated in (7). The simplest implementation of this model uses tissue physical properties that are held constant throughout the duration of the ablation. Typically, this model solves the electromagnetic problem using a steady-state solver, and then uses a fixed $Q$ as input for solution of the thermal model using a transient solver. Since tissue physical properties do not change for this model, there is no need to recompute $Q$ during the course of the ablation-that is, the coupling between the electromagnetic and thermal models is only in one direction. While such a model is relatively easy to implement, tissue temperatures obtained from such models are unrealistically large since they do not account for reduced tissue conductivity and permittivity at higher temperatures, as well as the significant heat sink associated with water vaporization [45]. Nevertheless, these models are an improvement over simple SAR models since they account for thermal conduction and blood perfusion, in the case of in vivo studies. Moreover, although tissue temperatures in the target region may be large, the extent of ablation zones measured using these models correlates well with ablation zones created in ex vivo experiments [45] .

Some studies use an experimentally measured SAR pattern to determine the heat source for the Pennes equation $[56,71]$. This approach yields excellent correlation with experimentally measured temperature profiles both ex vivo and in vivo, if peak tissue temperatures do not exceed $80^{\circ} \mathrm{C}$ [56]. However, at higher tissue temperatures, desiccation and water vaporization have a substantial impact on dielectric properties of tissue, thereby altering SAR pattern significantly.

\section{Comprehensive Models of Microwave Ablation}

The major shortcoming of models that solve the bioheat equation without incorporating varying tissue properties during the course of an ablation is that they compute unrealistically high tissue temperatures, which do not correlate well with experimental measurement. Analysis of changes in tissue dielectric properties during the course of the ablation provides an insight into why this occurs. Table 1 lists empirical formulae for tissue physical properties as a function of water content. As tissue water evaporates, water 


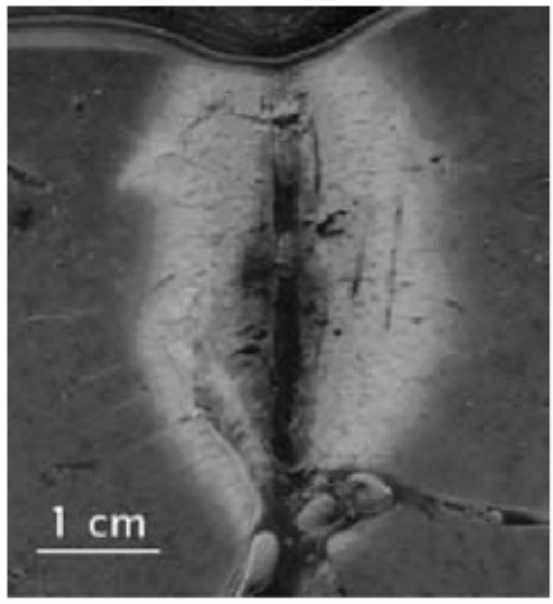

(a)

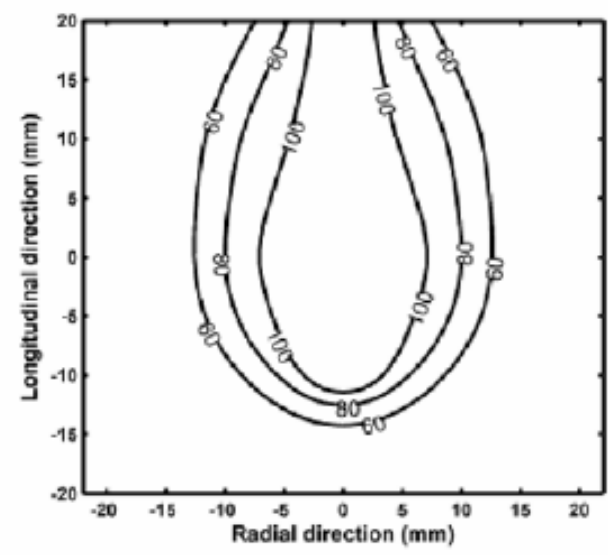

(b)

Fig. (2). Comparison of (a) ablation zone in created ex vivo bovine liver and (b) theoretically predicted temperature profiles using comprehensive theoretical simulation including dynamic changes in tissue properties. The $60{ }^{\circ} \mathrm{C}$ isotherm is in good agreement with visible boundary of coagulated tissue. (Reprinted with permission from [45], (C) 2007 IEEE).

content drops dramatically, resulting in tissue becoming less lossy. Thus, microwaves can penetrate further into tissue before getting absorbed. Consequently, less energy is absorbed in tissue closer to the antenna, and predicted tissue temperatures will be lower than compared to the case where tissue dielectric conductivity remains unchanged. Furthermore, there is significant heat loss associated with tissue water vaporization that is not accounted for in simplistic models.

Yang et al. $[45,73]$ presented a comprehensive computer model of high power microwave tissue ablation, incorporating dynamic changes in tissue dielectric and thermal properties, based on experimental measurements of tissue water content [39]. Their studies presented a mapping function to compute tissue water content from temperature. Since changes in physical properties of tissue due to changes in water content dominate any changes due to elevated temperature, physical properties of tissue were computed using the empirical formulae shown in Table $\mathbf{1}$. The comprehensive computer model provided good correlation with experimentally measured temperatures Fig. (2) shows an image of the ablation zone created in ex vivo bovine tissue, compared to simulated temperature profiles, taken from [45].

Although [45] is the most comprehensive model of microwave tissue ablation presented in the literature, it still has several shortcomings. First, the mapping between temperature and tissue water content only takes into account instantaneous temperature. Tissue water content is likely a function of the time-temperature history. More experiments need to be performed to determine if this mapping holds true in general. Second, the model assumes that evaporated tissue water diffuses from the target region and recondenses uniformly in tissue at lower temperatures. The model does not take into account any heat transfer that occurs between diffusing steam and bulk tissue. Lastly, the model does not include any consideration of dynamic changes in tissue blood perfusion during the course of ablation. Recent studies for low temperature MWA and RFA have shown that dynamic changes in tissue blood perfusion have a substantial impact on the size and shape of the predicted ablation zone $[56,57]$. Other models for high temperature MWA have accounted for tissue water vaporization, and associated changes in tissue thermal properties, only around the phase change temperature [47]. However, changes in the antenna radiation pattern after tissue water vaporization were not considered.

A comprehensive computer model for microwave tissue ablation, taking into account all changes in tissue properties critical to accurate modeling of tissue temperature and cellular damage, is yet to be developed. Fig. (3) illustrates the flow of operations for such a comprehensive model of microwave tissue ablation taking into account the complex physical phenomena occurring during high temperature ablation. Improved characterization of tissue properties would facilitate incorporation of these phenomena into existing models.

\section{CURRENT CHALLENGES AND RESEARCH TRENDS}

Computer modeling of MWA continues to be a prominent subject of research in the field of thermal tumor ablation. In this section, we review current challenges and research trends associated with theoretical modeling of microwave tissue ablation.

There have been few reports in the literature comparing theoretically predicted temperature profiles and tissue damage with in vivo experiments, especially for high temperature ablation. One of the main reasons for this has been the lack, until recently, of suitable techniques for spatial measurement of temperature during ablations. Most studies have measured temperature at discrete points using invasive thermal sensors. Recent developments in magnetic resonance imaging (MRI) have paved the way for accurate, high resolution, volumetric thermal imaging of tissue during ablations [74-76]. However, MRI machines necessitate the 
use of MRI compatible devices, which are not as readily available as simple copper-based coaxial cables. Moreover, techniques to overcome respiratory motion artifacts, often unavoidable in vivo, need to be developed [77]. Techniques for absolute temperature measurement using diagnostic ultrasound are also under development [78-80].

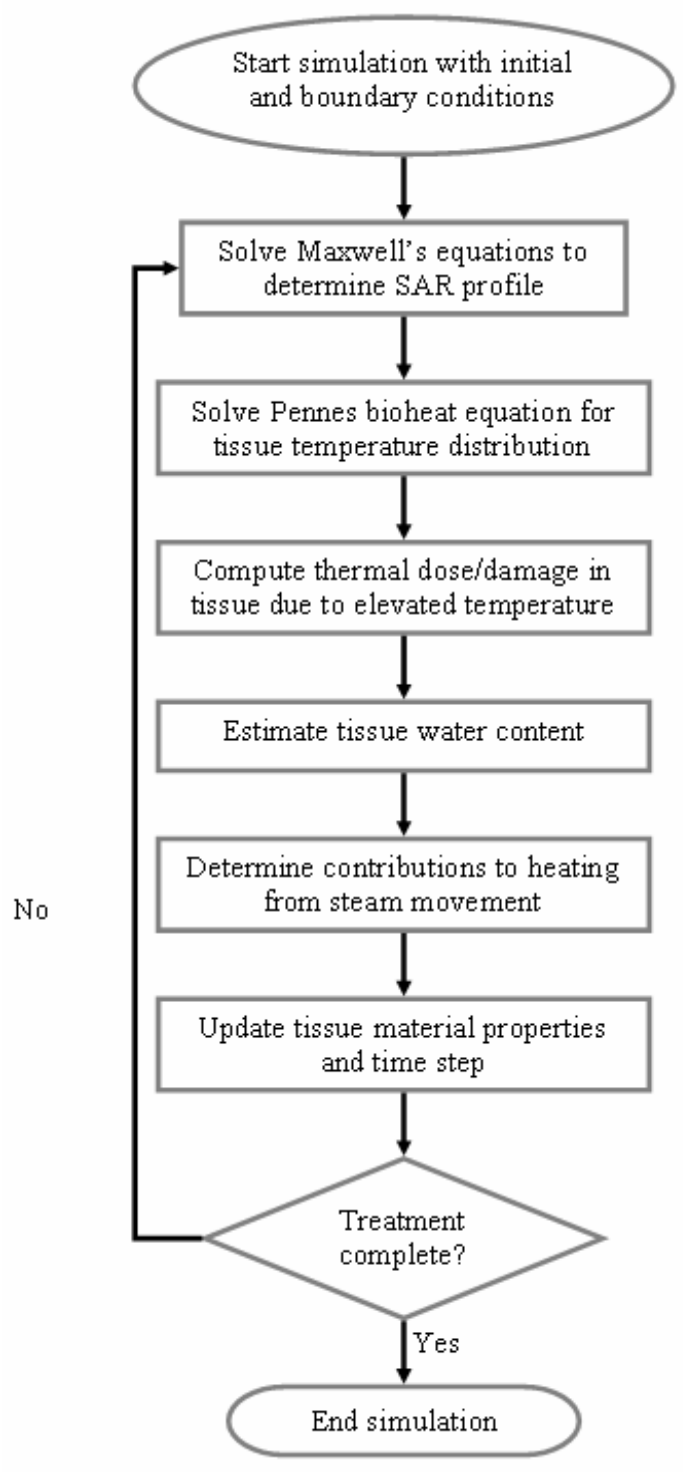

Fig. (3). Flow of operations for a comprehensive theoretical model for microwave tissue ablation. The treatment may be terminated based on a thermal coverage of a pre-specified region or after a given treatment time.

In addition to the theoretical models described in this paper, other computer models of microwave heating have been modeled for other applications. Models of heat and mass transfer in porous media have been developed for microwave heating with applications in food science [81, 82]. Modeling bulk liver tissue as a porous medium may make these models amenable for simulating microwave tissue ablation. However, applying these models necessitates measurements of several tissue physical properties that are currently unavailable. Moreover, the models are inherently complex and may not be suitable for quick evaluation in 3D geometries. Nevertheless, these models may provide an insight into the effects of steam heating, which has not been considered in models developed to date.

Blood flow in large, discrete vessels - macrovascular perfusion - is a significant heat sink that has substantial impact on size and shape of an ablation zone. One of the theoretical advantages of using microwave energy for ablation of highly perfused organs such as the liver and kidney is that it may be more effective for heating in the vicinity of large vessels. Some experimental studies have investigated the ability of ablating targets close to large blood vessels using microwave energy. Compared to lesions created by radiofrequency ablation, which tend to dip in near vessels, ablation zones created using microwave energy encompass the vessel. It is hypothesized that this may be due to heating effects of steam moving through the vessel as a path of least resistance. There are few reports of theoretical models to incorporate this behavior. Liu et al. [60] used a coupled fluid flow-thermal model to analyze performance of microwave ablation near an arterial bifurcation. However, their model does not include a high temperature treamtent and dynamic tissue changes. More comprehensive model taking into account dynamic tissue properties and steam heating are being explored. Impact of power deposition within the vessel (microwave energy absorbed by the vessel wall and blood) should also be considered. The impact of blood vessel cooling may be explored using a convective boundary condition on the vessel boundary, as has been done for RFA. A more accurate model would use the approach of [59] and include a coupled fluid flow model to explore the impact of various flow profiles on the ablation zone.

Antennas for microwave ablation have been developed to operate at frequencies of $915 \mathrm{MHz}$ and $2.45 \mathrm{GHz}$. At lower frequencies, penetration depth of microwaves is greater than at $2.45 \mathrm{GHz}$, which may lead to larger ablation zones. An in depth study of the optimal frequency for ablation has not been reported in the literature. Computer models are an ideal tool for exploring the tradeoff between different frequencies and selecting optimal operating frequencies for microwave ablation devices. They may also be used to explore modifications of device designs and treatment parameters for devices developed to treat other organs.

Theoretical models may also be used to explore different treatment delivery strategies. One of the advantages of microwave ablation is that higher tissue temperatures may be attained in vivo, leading to larger ablation zones. However, these higher temperatures may be undesirable or even dangerous in certain situations, for instance, when treating tumors near large blood vessels. Appropriate power modulation and control schemes are being explored to limit maximum tissue temperatures while ensuring complete target coverage. Theoretical models provide a quick and convenient method for evaluating various control algorithms.

The impact of inter-patient variations in tissue properties has not been studied in great detail for microwave ablation. Computer models are an excellent tool for performing such a study, as they allow quick evaluation of ablation quality for a wide range of parameters. A study of the effect of interpatient variation is of great importance as it helps determine the robustness of the treatment modality. Moreover, it may 
be used to determine how treatment parameters should be adjusted for different cases. Recently, dos Santos et al. [83] proposed a method to analyze the impact of random variations in tissue properties using the unscented transform and finite element simulations of radiofrequency ablation. Prakash et al. [69] considered interpatient variations in dielectric properties to optimize the design of an antenna robust to changes in dielectric properties of tissue. More extensive studies to analyze the full impact of varying tissue properties are warranted.

Computer models of thermal therapy taking into account patient specific anatomies are being developed for hyperthermia applications [84-86]. These models are a valuable resource for pre-planning treatments as well as for post-treatment assessment of cases. Treatment planning tools may help physicians performing ablation procedures determine parameters such as optimal antenna placements, power levels and treatment durations, a priori, for individual patients. These parameters may need to be adjusted during the course of the treatment given feedback from lesion monitoring technology e.g. temperature maps from MR thermometry. Currently, treatment planning for clinical RFA is heavily dependent on the physician performing the procedure. The development of accurate and fast computer models and technologies for monitoring growth of the ablation zone will pave the way for computerized treatment planning. Combining computer models capable of predicting temperature history and imaging/temperature scans of the anatomy being treated would allow physicians to get a better understanding of the chances of success before and during the treatment. Imprecise and suboptimal applicator positioning, especially for cases involving multiple overlapping ablation zones, has been highlighted as a potential contributor to increased recurrence rates for larger tumors. Combining imaging scans with computer models that highlight optimal treatment parameters will enable the use of automated applicator positioning, which may help reduce recurrence rates.

Patient specific models used for planning clinical treatments require accurate models that can be evaluated in near real time. The most comprehensive models of microwave ablation developed to date take several hours of computation time on a modern PC. Nonetheless, some studies have attempted to incorporate these models into interactive treatment planning systems. Zhai et al. [87] developed a patient-specific planning platform that allows surgeons to adjust the trajectory path for inserting an interstitial MWA antenna. The predicted zone of damage calculated from an FEM model is overlaid on the patient anatomy to help physicians determine optimal trajectories for inserting the applicator (Fig. 4). Some studies have considered using simplified models to improve computation time, at the expense of accuracy. Villard et al. [88] used a sphere-packing algorithm to determine optimal probe positioning for RFA. The size of spheres created by an ablation needle was determined from experimental data. While this method makes prediction of the size and shape of the ablation zone trivial, it is an overly simplistic model that does not take into account the physics of the phenomena involved. Moreover, it is easily affected by patient-specific conditions such as convective cooling to macrovascular perfusion. A more promising technique is the use of mathematical techniques to reduce the size of the computational problem, with minimal loss of accuracy. Altrogge et al. [89, 90] presented a multi-scale optimization approach for positioning electrodes during RFA. Modelorder reduction techniques have been used to speed up optimization of parameters for hyperthermia treatment [91].

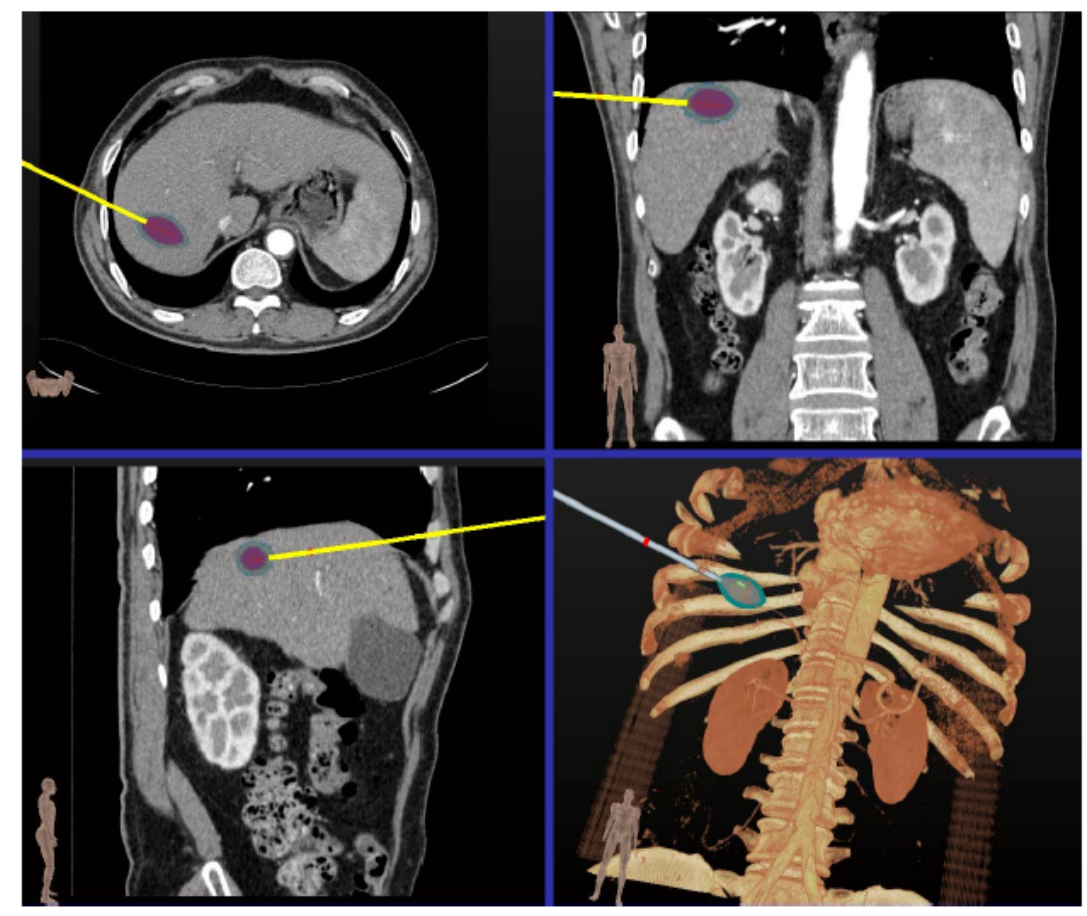

Fig. (4). Interactive planning suite for microwave ablation of liver tumors. Surgeons can evaluate different trajectory paths for inserting the antenna and observe the associated predicted ablation zone. (Reprinted with permission from [87]). 
The ability to predict the success of an ablation in the vicinity of large blood vessels using computer models would be very useful when selecting treatment parameters for ablating such tumors. Fuentes et al. [74] recently reported a treatment planning scheme for interstitial laser prostate therapy incorporating real-time model predictive control. MR thermometry taken during a treatment was used to calibrate parameters for the thermal model and determine optimal power levels throughout the course of the treatment. While this was an excellent example of the use of theoretical models for planning and control thermal ablation of tissue, it required a cluster of $\sim 20$ computers for fast model computation and optimization. Such computational facilities will likely be unavailable at most centers. Thus, substantial efforts to obtain models of acceptable accuracy within reasonable computational times must be explored to make patient-specific planning a reality.

\section{SUMMARY}

Microwave ablation is a thermal ablation mechanism capable of creating large ablation zones in shorter treatment times, compared to the current standard, radiofrequency ablation. Theoretical models predicting thermal profiles and tissue damage have played a substantial role in the development and understanding of devices used for MWA. Improved characterization of tissue properties and heat transfer mechanisms at elevated temperatures are required to improve models of high powered microwave ablation. Extensive validation of models in vivo, needs to be performed to verify their accuracy. Models including patient specific anatomies are being explored for pre-planning ablation therapies. Improvements in computation time will make these models more amenable for patient-specific treatment planning, similar to what is currently done for radiation therapy.

\section{REFERENCES}

[1] C. J. Diederich, "Thermal ablation and high-temperature thermal therapy: overview of technology and clinical implementation," Int. J. Hyperthermia, vol. 21, pp. 745-53, 2005.

[2] A. P. O'Rourke, D. Haemmerich, P. Prakash, M. C. Converse, D. M. Mahvi, and J. G. Webster, "Current status of liver tumor ablation devices," Expert. Rev. Med. Devices, vol. 4, pp. 523-37, 2007.

[3] R. W. Habash, R. Bansal, D. Krewski, and H. T. Alhafid, "Thermal therapy, Part III: ablation techniques," Crit. Rev. Biomed. Eng., vol. 35, pp. 37-121, 2007.

[4] T. C. Winter, P. F. Laeseke, and F. T. Lee, Jr., "Focal tumor ablation: a new era in cancer therapy," Ultrasound Q., vol. 22, pp. 195-217, 2006.

[5] S. Garrean, J. Hering, A. Saied, W. S. Helton, and N. J. Espat, "Radiofrequency ablation of primary and metastatic liver tumors: a critical review of the literature," Am. J. Surg., vol. 195, pp. 508-20, 2008.

[6] T. Mala, "Cryoablation of liver tumours -- a review of mechanisms, techniques and clinical outcome," Minim. Invasive. Ther. Allied Technol., vol. 15, pp. 9-17, 2006.

[7] C. J. Simon, D. E. Dupuy, and W. W. Mayo-Smith, "Microwave ablation: principles and applications," Radiographics, vol. 25 (Suppl 1), pp. S69-83, 2005.

[8] C. L. Brace, "Microwave ablation technology: what every user should know," Curr. Probl. Diagn. Radiol., vol. 38, pp. 61-7, 2009.

[9] P. Liang, Y. Wang, D. Zhang, X. Yu, Y. Gao, and X. Ni, "Ultrasound guided percutaneous microwave ablation for small renal cancer: initial experience," J. Urol., vol. 180, pp. 844-8, discussion 848, 2008.

[10] P. E. Clark, R. D. Woodruff, R. J. Zagoria, and M. C. Hall, "Microwave ablation of renal parenchymal tumors before nephrectomy: phase I study," Am. J. Roentgenol., vol. 188, pp. 1212-4, 2007.

[11] C. L. Brace, J. L. Hinshaw, P. F. Laeseke, L. A. Sampson, and F. T. Lee, Jr., "Pulmonary Thermal Ablation: Comparison of Radiofrequency and Microwave Devices by Using Gross Pathologic and CT Findings in a Swine Model," Radiology, vol. 251, no. 3, pp. 705-11, 2009.

[12] R. A. McTaggart, and D. E. Dupuy, "Thermal ablation of lung tumors," Tech. Vasc. Interv. Radiol., vol. 10, pp. 102-13, 2007.

[13] E. J. Wasser, and D. E. Dupuy, "Microwave ablation in the treatment of primary lung tumors," Semin Respir. Crit. Care Med., vol. 29, pp. 384-94, 2008.

[14] T. Moser, X. Buy, G. Goyault, C. H. Tok, F. Irani, and A. Gangi, "[Image-guided ablation of bone tumors: review of current techniques]," J. Radiol., vol. 89, pp. 461-71, 2008.

[15] C. L. Brace, "Radiofrequency and microwave ablation of the liver, lung, kidney, and bone: what are the differences?," Curr. Probl. Diagn. Radiol., vol. 38, pp. 135-43, 2009.

[16] L. Wagrell, T. Schelin, M. B. Bolmsjo, and A. Mattiasson, "Aspects on transurethral microwave thermotherapy of benign prostatic hyperplasia," Tech. Urol., vol. 6, pp. 251-5, 2000.

[17] M. B. Bolmsjo, and T. Vrba, "Microwave applicators for thermotherapy of benign prostatic hyperplasia: a primer," Tech. Urol., vol. 6, pp. 245-50, 2000.

[18] W. A. Kaiser, S. O. Pfleiderer, and P. A. Baltzer, "MRI-guided interventions of the breast," J. Magn. Reson. Imaging, vol. 27, pp. 347-55, 2008.

[19] G. M. Comas, Y. Imren, and M. R. Williams, "An overview of energy sources in clinical use for the ablation of atrial fibrillation," Semin. Thorac. Cardiovasc. Surg., vol. 19, pp. 16-24, 2007.

[20] H. Kroeze, J. B. Van de Kamer, A. A. De Leeuw, and J. J. Lagendijk, "Regional hyperthermia applicator design using FDTD modelling," Phys. Med. Biol., vol. 46, pp. 1919-35, 2001.

[21] P. Wust, B. Hildebrandt, G. Sreenivasa, B. Rau, J. Gellermann, H. Riess, R. Felix, and P. M. Schlag, "Hyperthermia in combined treatment of cancer," Lancet Oncol., vol. 3, pp. 487-97, 2002.

[22] L. Hamada, K. Saito, H. Yoshimura, and K. Ito, "Dielectric-loaded coaxial-slot antenna for interstitial microwave hyperthermia: longitudinal control of heating patterns," Int. J. Hyperthermia, vol. 16, pp. 219-29, 2000.

[23] K. Ito, K. Saito, H. Yoshimura, Y. Aoyagi, and H. Horita, "Coaxial-slot antenna for interstitial microwave thermal therapy and its application to clinical trial," Conf. Proc. IEEE Eng. Med. Biol. Soc., vol. 4, pp. 2526-9, 2004.

[24] J. C. Lin, and Y. J. Wang, "The cap-choke catheter antenna for microwave ablation treatment," IEEE Trans. Biomed. Eng., vol. 43, pp. 657-60, 1996.

[25] D. Yang, J. M. Bertram, M. C. Converse, A. P. O'Rourke, J. G. Webster, S. C. Hagness, J. A. Will, and D. M. Mahvi, "A floating sleeve antenna yields localized hepatic microwave ablation," IEEE Trans. Biomed. Eng., vol. 53, pp. 533-7, 2006.

[26] C. L. Brace, P. F. Laeseke, L. A. Sampson, T. M. Frey, D. W. van der Weide, and F. T. Lee, Jr., "Microwave ablation with a single small-gauge triaxial antenna: in vivo porcine liver model," Radiology, vol. 242, pp. 435-40, 2007.

[27] C. L. Brace, P. F. Laeseke, D. W. van der Weide, and F. T. Lee, "Microwave Ablation With a Triaxial Antenna: Results in ex vivo Bovine Liver," IEEE Trans. Microw. Theory Tech., vol. 53, pp. 215-220, 2005.

[28] J. M. Bertram, D. Yang, M. C. Converse, J. G. Webster, and D. M. Mahvi, "A review of coaxial-based interstitial antennas for hepatic microwave ablation," Crit. Rev. Biomed. Eng., vol. 34, pp. 187213, 2006.

[29] S. A. Sapareto, and W. C. Dewey, "Thermal dose determination in cancer therapy," Int. J. Radiat. Oncol. Biol. Phys., vol. 10, pp. 787800, 1984.

[30] H. Rhim, S. N. Goldberg, G. D. Dodd, 3rd, L. Solbiati, H. K. Lim, M. Tonolini, and O. K. Cho, "Essential techniques for successful radio-frequency thermal ablation of malignant hepatic tumors," Radiographics, vol. 21 Spec No, pp. S17-35; discussion S36-9, 2001.

[31] W. C. Dewey, "Arrhenius relationships from the molecule and cell to the clinic," Int. J. Hyperthermia, vol. 10, pp. 457-83, 1994.

[32] S. Gabriel, R. W. Lau, and C. Gabriel, "The dielectric properties of biological tissues: III. Parametric models for the dielectric spectrum of tissues," Phys. Med. Biol., vol. 41, pp. 2271-93, 1996. 
[33] F. A. Duck, Physical properties of tissue: a comprehensive reference book. London: 1990.

[34] P. R. Stauffer, F. Rossetto, M. Prakash, D. G. Neuman, and T. Lee, "Phantom and animal tissues for modelling the electrical properties of human liver," Int. J. Hyperthermia, vol. 19, pp. 89-101, 2003.

[35] A. P. O'Rourke, M. Lazebnik, J. M. Bertram, M. C. Converse, S. C. Hagness, J. G. Webster, and D. M. Mahvi, "Dielectric properties of human normal, malignant and cirrhotic liver tissue: in vivo and ex vivo measurements from 0.5 to $20 \mathrm{GHz}$ using a precision openended coaxial probe," Phys. Med. Biol., vol. 52, pp. 4707-19, 2007.

[36] M. Lazebnik, M. C. Converse, J. H. Booske, and S. C. Hagness, "Ultrawideband temperature-dependent dielectric properties of animal liver tissue in the microwave frequency range," Phys. Med. Biol., vol. 51, pp. 1941-55, 2006.

[37] C. L. Brace, "Temperature-dependent dielectric properties of liver tissue measured during thermal ablation: toward an improved numerical model," Conf. Proc. IEEE Eng. Med. Biol. Soc., vol. 2008, pp. 230-3, 2008.

[38] D. Yang, M. C. Converse, D. M. Mahvi, and J. G. Webster, "Measurement and analysis of tissue temperature during microwave liver ablation," IEEE Trans. Biomed. Eng., vol. 54, pp. 150-5, 2007.

[39] J. L. Schepps, and K. R. Foster, "The UHF and microwave dielectric properties of normal and tumour tissues: variation in dielectric properties with tissue water content," Phys. Med. Biol., vol. 25, pp. 1149-59, 1980.

[40] H. H. Pennes, "Analysis of tissue and arterial blood temperatures in the resting human forearm," J. Appl. Physiol., vol. 85, pp. 5-34, 1948.

[41] D. Haemmerich, I. dos Santos, D. J. Schutt, J. G. Webster, and D. M. Mahvi, "In vitro measurements of temperature-dependent specific heat of liver tissue," Med. Eng. Phys., vol. 28, pp. 194-7, 2006.

[42] D. Haemmerich, D. J. Schutt, I. dos Santos, J. G. Webster, and D. M. Mahvi, "Measurement of temperature-dependent specific heat of biological tissues," Physiol. Meas., vol. 26, pp. 59-67, 2005.

[43] G. Shafirstein, W. Baumler, M. Lapidoth, S. Ferguson, P. E. North, and M. Waner, "A new mathematical approach to the diffusion approximation theory for selective photothermolysis modeling and its implication in laser treatment of port-wine stains," Lasers Surg. Med., vol. 34, pp. 335-47, 2004.

[44] D. Yang, M. C. Converse, D. M. Mahvi, and J. G. Webster, "Expanding the bioheat equation to include tissue internal water evaporation during heating," IEEE Trans. Biomed. Eng., vol. 54, pp. 1382-8, 2007.

[45] E. Sparrow, and J. Abraham, "A simulation of gas-based, endometrial-ablation therapy," Ann. Biomed. Eng., vol. 36, pp. 17183, 2008.

[46] J. P. Abraham, E. M. Sparrow, and S. Ramadhyani, "Numerical simulation of a BPH thermal therapy--a case study involving TUMT," J. Biomech. Eng., vol. 129, pp. 548-57, 2007.

[47] A. Bhattacharya, and R. L. Mahajan, "Temperature dependence of thermal conductivity of biological tissues," Physiol. Meas., vol. 24, pp. 769-83, 2003.

[48] J. W. Valvano, J. R. Cochran, and K. R. Diller, "ThermalConductivity and Diffusivity of Biomaterials Measured with SelfHeated Thermistors," Int. J. Thermophys., vol. 6, pp. 301-311, 1985.

[49] S. Weinbaum, and L. M. Jiji, "A new simplified bioheat equation for the effect of blood flow on local average tissue temperature," $J$. Biomech. Eng., vol. 107, pp. 131-9, 1985.

[50] H. Arkin, L. X. Xu, and K. R. Holmes, "Recent developments in modeling heat transfer in blood perfused tissues," IEEE Trans. Biomed. Eng., vol. 41, pp. 97-107, 1994.

[51] A. Kotte, G. van Leeuwen, J. de Bree, J. van der Koijk, H. Crezee, and J. Lagendijk, "A description of discrete vessel segments in thermal modelling of tissues," Phys. Med. Biol., vol. 41, pp. 86584, 1996.

[52] J. W. Valvano, "Bioheat transfer," in Encyclopedia of Medical Devices and Instrumentation, J. G. Webster, Ed., Second ed: John Wiley and Sons, Inc., 2006.

[53] S. L. Brown, J. W. Hunt, and R. P. Hill, "Differential thermal sensitivity of tumour and normal tissue microvascular response during hyperthermia," Int. J. Hyperthermia, vol. 8, pp. 501-14, 1992.
[54] M. van Vulpen, B. W. Raaymakers, A. A. de Leeuw, J. B. van de Kamer, R. J. van Moorselaar, M. G. Hobbelink, J. J. Battermann, and J. J. Lagendijk, "Prostate perfusion in patients with locally advanced prostate carcinoma treated with different hyperthermia techniques," J. Urol., vol. 168, pp. 1597-602, 2002.

[55] X. He, S. McGee, J. E. Coad, F. Schmidlin, P. A. Iaizzo, D. J. Swanlund, S. Kluge, E. Rudie, and J. C. Bischof, "Investigation of the thermal and tissue injury behaviour in microwave thermal therapy using a porcine kidney model," Int. J. Hyperthermia, vol. 20, pp. 567-93, 2004.

[56] D. J. Schutt, and D. Haemmerich, "Effects of variation in perfusion rates and of perfusion models on radiofrequency tumor ablation," Med. Phys., vol. 35, no. 8, pp. 3462-70, 2008.

[57] D. Haemmerich, A. W. Wright, D. M. Mahvi, F. T. Lee, Jr., and J. G. Webster, "Hepatic bipolar radiofrequency ablation creates coagulation zones close to blood vessels: a finite element study," Med. Biol. Eng. Comput., vol. 41, pp. 317-23, 2003.

[58] I. dos Santos, D. Haemmerich, S. Pinheiro Cda, and A. F. da Rocha, "Effect of variable heat transfer coefficient on tissue temperature next to a large vessel during radiofrequency tumor ablation," Biomed. Eng. Online, vol. 7, pp. 21, 2008.

[59] Y. J. Liu, A. K. Qiao, Q. Nan, and X. Y. Yang, "Thermal characteristics of microwave ablation in the vicinity of an arterial bifurcation," Int. J. Hyperthermia, vol. 22, pp. 491-506, 2006.

[60] J. Pearce, "Relationship between Arrhenius models of thermal damage and the CEM 43 thermal dose," presented at Proceedings of SPIE, 2009.

[61] W. C. Dewey, and C. J. Diederich, "Hyperthermia classic commentary: 'Arrhenius relationships from the molecule and cell to the clinic' by William Dewey, Int. J. Hyperthermia, 10:457-483, 1994," Int J Hyperthermia, vol. 25, pp. 21-4, 2009.

[62] F. Henriques, "Studies of thermal injury. V. The predictability and the significance of thermally induced rate processes leading to irreversible epidermal injury," Arch. Pathol., vol. 43, pp. 489-502, 1947.

[63] A. Moritz, and F. Henriques Jr, "Studies of Thermal Injury: II. The Relative Importance of Time and Surface Temperature in the Causation of Cutaneous Burns*," Am. J. Pathol., vol. 23, pp. 695, 1947.

[64] S. Thomsen, J. A. Pearce, and W. F. Cheong, "Changes in birefringence as markers of thermal damage in tissues," IEEE Trans. Biomed. Eng., vol. 36, pp. 1174-9, 1989.

[65] G. Schueller, J. Kettenbach, R. Sedivy, A. Stift, J. Friedl, M. Gnant, and J. Lammer, "Heat shock protein expression induced by percutaneous radiofrequency ablation of hepatocellular carcinoma in vivo," Int. J. Oncol., vol. 24, pp. 609-13, 2004.

[66] J. M. Bertram, D. Yang, M. C. Converse, J. G. Webster, and D. M. Mahvi, "Antenna design for microwave hepatic ablation using an axisymmetric electromagnetic model," Biomed. Eng. Online, vol. 5, p. $15,2006$.

[67] S. Hagness, and A. Taflove, "Computational electrodynamics: The finite-difference time-domain method," Norwood, MA: Artech House, 2000

[68] P. Prakash, G. Deng, M. C. Converse, J. G. Webster, D. M. Mahvi, and M. C. Ferris, "Design optimization of a robust sleeve antenna for hepatic microwave ablation," Phys. Med. Biol., vol. 53, pp. 1057-69, 2008

[69] W. Schramm, D. Yang, and D. Haemmerich, "Contribution of direct heating, thermal conduction and perfusion during radiofrequency and microwave ablation," Conf. Proc. IEEE Eng. Med. Biol. Soc., vol. 1, pp. 5013-6, 2006.

[70] Y. Liu, X. Yang, Q. Nan, J. Xiao, and L. Li, "Phantom experimental study on microwave ablation with a water-cooled antenna," Int. J. Hyperthermia, vol. 23, pp. 381-6, 2007.

[71] S. Pisa, M. Cavagnaro, P. Bernardi, and J. C. Lin, "A 915-MHz antenna for microwave thermal ablation treatment: physical design, computer modeling and experimental measurement," IEEE Trans. Biomed. Eng., vol. 48, pp. 599-601, 2001.

[72] D. Yang, "Measurements, antenna design and advanced computer modeling for microwave tissue ablation," in Biomedical Engineering. Madison: University of Wisconsin, 2006.

[73] D. Fuentes, J. T. Oden, K. R. Diller, J. D. Hazle, A. Elliott, A. Shetty, and R. J. Stafford, "Computational modeling and real-time control of patient-specific laser treatment of cancer," Ann. Biomed. Eng., vol. 37, pp. 763-82, 2009. 
[74] B. D. de Senneville, C. Mougenot, B. Quesson, I. Dragonu, N. Grenier, and C. T. Moonen, "MR thermometry for monitoring tumor ablation," Eur. Radiol., vol. 17, pp. 2401-10, 2007.

[75] V. Rieke and K. Butts Pauly, "MR thermometry," J. Magn. Reson. Imaging, vol. 27, pp. 376-90, 2008.

[76] S. Hey, G. Maclair, B. D. de Senneville, M. Lepetit-Coiffe, Y. Berber, M. O. Kohler, B. Quesson, C. T. Moonen, and M. Ries, "Online correction of respiratory-induced field disturbances for continuous MR-thermometry in the breast," Magn. Reson. Med., vol. 61, no. 6, pp. 1494-9, 2009.

[77] M. J. Daniels, T. Varghese, E. L. Madsen, and J. A. Zagzebski, "Non-invasive ultrasound-based temperature imaging for monitoring radiofrequency heating-phantom results," Phys. Med. Biol., vol. 52, pp. 4827-43, 2007.

[78] M. J. Daniels, J. Jiang, and T. Varghese, "Ultrasound simulation of real-time temperature estimation during radiofrequency ablation using finite element models," Ultrasonics, vol. 48, pp. 40-55, 2008.

[79] D. Liu, A. Shrestha, R. Visaria, J. Bischof, and E. S. Ebbini, "Noninvasive temperature estimation using diagnostic ultrasound: In vivo results," J. Acoust. Soc. Am., vol. 123, pp. 3000, 2008.

[80] A. K. Datta, "Porous media approaches to studying simultaneous heat and mass transfer in food processes. I: Problem formulations," J. Food Eng., vol. 80, pp. 80-95, 2007.

[81] A. K. Datta, "Porous media approaches to studying simultaneous heat and mass transfer in food processes. II: Property data and representative results," J. Food Eng., vol. 80, pp. 96-110, 2007.

[82] I. Dos Santos, D. Haemmerich, D. Schutt, A. F. da Rocha, and L. R. Menezes, "Probabilistic finite element analysis of radiofrequency liver ablation using the unscented transform," Phys. Med. Biol., vol. 54, pp. 627-40, 2009.

[83] J. J. Lagendijk, "Hyperthermia treatment planning," Phys. Med. Biol., vol. 45, pp. R61-76, 2000.
[84] H. Kroeze, J. B. van de Kamer, A. A. de Leeuw, M. Kikuchi, and J. J. Lagendijk, "Treatment planning for capacitive regional hyperthermia," Int. J. Hyperthermia, vol. 19, pp. 58-73, 2003.

[85] C. A. Van den Berg, L. W. Bartels, A. A. De Leeuw, J. J. Lagendijk, and J. B. Van de Kamer, "Experimental validation of hyperthermia SAR treatment planning using MR B1+ imaging," Phys. Med. Biol., vol. 49, pp. 5029-42, 2004.

[86] W. Zhai, J. Xu, Y. Zhao, Y. Song, L. Sheng, and P. Jia, "Preoperative surgery planning for percutaneous hepatic microwave ablation," Med. Image Comput. Comput. Assist. Interv. Int. Conf. Med. Image Comput. Comput. Assist. Interv., vol. 11, pp. 569-77, 2008.

[87] C. Villard, L. Soler, and A. Gangi, "Radiofrequency ablation of hepatic tumors: simulation, planning, and contribution of virtual reality and haptics," Comput. Methods Biomech. Biomed. Engin., vol. 8, pp. 215-27, 2005 .

[88] I. Altrogge, T. Kroger, T. Preusser, C. Buskens, P. L. Pereira, D. Schmidt, A. Weihusen, and H. O. Peitgen, "Towards optimization of probe placement for radio-frequency ablation," Med. Image Comput. Comput. Assist. Interv. Int. Conf. Med. Image Comput. Comput. Assist. Interv., vol. 9, pp. 486-93, 2006.

[89] I. Altrogge, T. Preusser, T. Kroger, C. Buskens, P. L. Pereira, D. Schmidt, and H. O. Peitgen, "Multiscale optimization of the probe placement for radiofrequency ablation," Acad. Radiol., vol. 14, pp. 1310-24, 2007

[90] J. K. Potocki, and H. S. Tharp, "Reduced-order modeling for hyperthermia control," IEEE Trans. Biomed. Eng., vol. 39, pp. 1265-73, 1992.

[91] M. E. Kowalski and J. M. Jin, "Model-order reduction of nonlinear models of electromagnetic phased-array hyperthermia," IEEE Trans. Biomed. Eng., vol. 50, pp. 1243-54, 2003.

(C) Punit Prakash; Licensee Bentham Open.

This is an open access article licensed under the terms of the Creative Commons Attribution Non-Commercial License (http://creativecommons.org/licenses/by-nc/3.0/) which permits unrestricted, non-commercial use, distribution and reproduction in any medium, provided the work is properly cited. 